\title{
Spin Characters of Hyperoctahedral Wreath Products
}

\author{
Xiaoli $\mathrm{Hu}$ and Naihuan Jing*
}

\begin{abstract}
The irreducible spin character values of the wreath products of the hyperoctahedral groups with an arbitrary finite group are determined.
\end{abstract}

\section{Introduction}

Irreducible spin characters of the symmetric group $S_{n}$ were studied by Schur [17] as one of the important examples in representation theory. Schur first derived a Frobenius-type formula for the nontrivial spin character values at conjugacy classes corresponding to partitions with odd integer parts in terms of Schur's $Q$-functions, which is similar to the case of the symmetric group. Furthermore, Schur showed that beyond the Frobenius-type formula, there are other spin character values on special conjugacy classes corresponding to (odd) strict partitions, which are not given by his Q-functions but derived via his new twisted tensor product of basic spin representations.

After Schur's classic paper there have been quite a number of papers devoted to the spin groups and in particular, hyperoctahedral spin groups and their generalizations. Morris gave an alternative description of the twisted tensor product [12 and derived an iterative formula for Schur's $Q$-functions. Later Morris also studied double covering groups of Weyl groups following Schur's theory [13. Sergeev [16 showed that representation theory of the twisted hyperoctahedral group $\widetilde{H}_{n}$ is similar to that of the spin group $\widetilde{S}_{n}$ (cf. [15, 10]), and proved that Schur's $Q$-functions also served as generating functions for some irreducible spin supercharacters of the hyperoctahedral groups. Finally Yamaguchi [19] clarified this relationship and established an equivalence between the twisted group algebra of the symmetric group and the queer Lie superalgebra.

2010 Mathematics Subject Classification. Primary: 20C25; Secondary: 20C30, 20B30, $20 \mathrm{C} 15$.

Key words and phrases. hyperoctahedral groups; wreath products; spin characters.

Supported by NSFC grants 11271138, 11426116 and Simons Foundation grant 198129.

* Corresponding Author. 
For the wreath product $\widetilde{\Gamma}_{n}=\Gamma \imath \widetilde{S}_{n}$ of a finite group $\Gamma$ by the spin symmetric group $\widetilde{S}_{n}$, most part of the spin character table was determined by Frenkel, Wang and the second author using the vertex operator calculus 7]. The remaining part was completed by the authors [4, 5] using Clifford algebras. In 2002, Wang and the second author [7] generalized the method to study the semi-direct product $\widetilde{H} \Gamma_{n}$ of the spin hyperoctahedral group $\widetilde{H}_{n}$ by a finite group $\Gamma$. The status of the character theory can be easily described as follows. If we denote by $\Gamma_{*}$ (resp. $\Gamma^{*}$ ) the set of conjugacy classes (resp. irreducible characters) of $\Gamma$, then the irreducible spin characters of $\widetilde{H} \Gamma_{n}$ are determined by pairs of the strict partition-valued functions on $\Gamma^{*}$ and the spin character values at the even conjugacy classes are provided by vertex operators in [7]. However, the spin character values at odd strict partitionvalued functions of $\Gamma_{*}$ are still unknown.

This paper aims to give the complete spin character table of the semidirect product $\widetilde{H} \Gamma_{n}$, which include projective characters of various Weyl groups and their wreath products. Part of the motivation is to extend the previous type $A$ theory (the character theory of $\widetilde{\Gamma}_{n}$ ) to type $B$ or $D$. The following table shows the situation with the new case. Here $\mathcal{S P}_{n}^{1}\left(\Gamma_{*}\right)$ (resp. $\mathcal{S} \mathcal{P}_{n}^{1}\left(\Gamma^{*}\right)$ ) denotes the set of odd strict partition-valued functions on $\Gamma_{*}$ (resp. $\left.\Gamma^{*}\right)$ and $\mathcal{O} \mathcal{P}_{n}\left(\Gamma_{*}\right)$ (resp. $\left.\mathcal{O} \mathcal{P}_{n}\left(\Gamma^{*}\right)\right)$ denotes the set of odd integer partitionvalued functions on $\Gamma_{*}\left(\right.$ resp. $\left.\Gamma^{*}\right)$, where $\Gamma_{*}\left(\right.$ resp. $\left.\Gamma^{*}\right)$ denotes the set of conjugacy classes (resp. irreducible characters) of the finite group $\Gamma$. The unknown part of the character values so far is denoted by a question mark. Note that there are two parities for spin hyperoctahedral wreath products and parition-valued functions, it happens that they are compatible for split conjugacy classes (see Prop. 3.1).

\begin{tabular}{|c|c|c|}
\hline \multirow{2}{*}{ Spin chars $\backslash$ Conj. classes } & $\rho \in \mathcal{O} \mathcal{P}_{n}\left(\Gamma_{*}\right)$ (even) & $\rho \in \mathcal{S P}_{n}^{1}\left(\Gamma_{*}\right)$ (odd) \\
\cline { 2 - 3 } & & \\
\hline$\nu \in \mathcal{S} \mathcal{P}_{n}\left(\Gamma^{*}\right)$ & & \\
$l(\nu)$ even & vertex operators & \\
$\chi_{\nu}$ & & $?$ \\
\hline $\begin{array}{c}\mathcal{S} \mathcal{P}_{n}\left(\Gamma^{*}\right) \\
l(\nu) \text { odd } \\
\chi_{\nu}^{ \pm}\end{array}$ & vertex operators & \\
\hline
\end{tabular}

TABLE 1. Spin character table of $\widetilde{H} \Gamma_{n}$

The spin characters computable by vertex operators are actually character values that come from certain supermodules of the wreath products of hyperoctahedral groups. It turns out that the characters values at conjugacy classes indexed by partition-valued functions with odd integer parts 
are the only non-zero values for certain modules, which then give the first part shown in the table.

Our method to uncover the other spin character values is a detailed analysis of difference characters for the ordinary modules and also how they are related with the characters of the supermodules. We generalize Józefiak's supermodule approach [8, 10 to study spin representations of $\widetilde{H} \Gamma_{n}$ and compute the associate spin characters at the odd strict conjugacy classes. While we take the advantage of the similarity between the representation theories of the wreath products and their hyperoctahedral counterparts, we also need to single out the difference in order to determine the nontrivial spin character values of the latter groups. Moreover, there is also another new point for hyperoctahedral wreath products. Previous work has been emphasizing their representations as a super theory, however in the current situation one needs to disengage the super theory to get their ordinary counterpart.

\section{The spin hyperoctahedral groups}

Let $S_{n}$ be the symmetric group on $n$ letters for $n \geq 4$. The hyperoctahedral group $H_{n}:=\mathbb{Z}_{2} \nmid S_{n}$ is generated by transpositions $s_{i}=(i, i+1)(1 \leq$ $i \leq n-1)$ and involutions $b_{j}(1 \leq j \leq n)$ with the relations

$$
\begin{array}{r}
s_{i}^{2}=1,\left(s_{i} s_{i+1}\right)^{3}=1,\left(s_{i} s_{j}\right)^{2}=1 \text { for }|i-j| \geq 2, \\
s_{i} b_{i}=b_{i+1} s_{i}, s_{i} b_{j}=b_{j} s_{i} \text { for } j \neq i, i+1, \\
b_{j}^{2}=1, b_{i} b_{j}=b_{j} b_{i} \text { for } i \neq j .
\end{array}
$$

We denote by $\widetilde{H}_{n}$ the group generated by $s_{i}(1 \leq i \leq n-1)$ and $a_{j}(1 \leq$ $j \leq n$ ) and $z$ with the following relations:

$$
\begin{array}{r}
s_{i}^{2}=1,\left(s_{i} s_{i+1}\right)^{3}=1, \quad\left(s_{i} s_{j}\right)^{2}=1 \text { for }|i-j| \geq 2, \\
s_{i} a_{i}=a_{i+1} s_{i}, \quad s_{i} a_{j}=a_{j} s_{i} \text { for } j \neq i, i+1, \\
a_{j}^{2}=z, \quad a_{i} a_{j}=z a_{j} a_{i} \text { for } i \neq j,
\end{array}
$$

where $z$ belong to the center and $z^{2}=1$.

Note that $\widetilde{H}_{n}$ is a semidirect product of the symmetric group $S_{n}=$ $\left\langle s_{i} \mid 1 \leq i \leq n-1\right\rangle$ and the 2-group generated by $a_{j}$ subject to the relations (2.4). Furthermore, $\widetilde{H}_{n}$ is a central extension of $H_{n}=\mathbb{Z}_{2}^{n} \rtimes S_{n}$ through

$$
1 \longrightarrow\langle z\rangle \hookrightarrow \widetilde{H}_{n} \stackrel{\theta_{n}}{\longrightarrow} H_{n} \longrightarrow 1
$$

where $\theta_{n}$ is the group homomorphism sending $s_{i}$ to $(i, i+1)$ for $1 \leq i \leq n-1$, $a_{j}$ to $b_{j}$ for $1 \leq i \leq n$, and $z$ to 1 . The group $\widetilde{H}_{n}$ is $\mathbb{Z}_{2}$-graded with the parity defined by $p\left(a_{i}\right)=1$ and $p\left(s_{i}\right)=p(z)=0$. With this parity the group algebra $\mathbb{C}\left(\widetilde{H}_{n}\right)$ becomes a superalgebra. 
The spin symmetric group $\widetilde{S}_{n}$ is the finite group generated by $t_{i}(1 \leq$ $i \leq n-1)$ and the central element $z$ with the relations

$$
t_{i}^{2}=z, \quad z^{2}=1, \quad\left(t_{i} t_{i+1}\right)^{3}=1, \quad t_{i} t_{j}=z t_{j} t_{i} \text { for }|i-j| \geq 2 .
$$

For each $n>1$, let $\mathscr{A}_{n}$ denote the algebra generated by $\varsigma_{i}(1 \leq i \leq n-1)$ and satisfy the following relations:

$$
\begin{aligned}
\varsigma_{i}^{2}=-1 & (1 \leq i \leq n-1), \\
\left(\varsigma_{i} \varsigma_{j}\right)^{2}=-1 & (|i-j| \geq 2), \\
\left(\varsigma_{i} \varsigma_{i+1}\right)^{3}=-1 & (1 \leq i \leq n-2) .
\end{aligned}
$$

The algebra $\mathscr{A}_{n}$ is also a superalgebra under the parity that $d\left(\varsigma_{i}\right)=1(1 \leq$ $i \leq n-1$ ). In fact $\mathscr{A}_{n}$ is isomorphic (as a superalgebra) to the twisted group algebra $\mathbb{C}\left(\widetilde{S}_{n}\right) /(1+z)$ via $\varsigma_{i} \mapsto t_{i}[\mathbf{1 7}]$.

For $n>1$, let $\mathscr{B}_{n}$ be the algebra generated by $\omega_{i}(0 \leq i \leq n-1)$ satisfying

$$
\begin{aligned}
& \omega_{i}^{2}=1(0 \leq i \leq n-1), \quad\left(\omega_{i} \omega_{i+1}\right)^{3}=1(1 \leq i \leq n-2), \\
& \left(\omega_{i} \omega_{j}\right)^{2}=1(|i-j| \geq 2), \quad\left(\omega_{0} \omega_{1}\right)^{4}=-1 .
\end{aligned}
$$

The algebra $\mathscr{B}_{n}$ is the group algebra of a double covering group of the Coxeter group $H_{n}$ and also a superalgebra by defining $d\left(\omega_{0}\right)=1$ and $d\left(\omega_{i}\right)=$ 0 for $1 \leq i \leq n-1$. Moreover $\mathscr{B}_{n} \cong \mathbb{C}\left[\widetilde{H}_{n}\right] /(1+z)$ via $\omega_{i} \mapsto t_{i}(1 \leq i \leq$ $n-1)$ and $\tau_{i}=\omega_{i-1} \cdots \omega_{1} \omega_{0} \omega_{1} \cdots \omega_{i-1} \mapsto a_{i}$ for $i=1, \ldots, n$. In particular, $\omega_{0} \equiv \tau_{1}$.

Let $\mathscr{C}_{n}$ be the Clifford algebra generated by $\xi_{i}(i=1, \ldots, n)$ subject to the relations

$$
\xi_{i}^{2}=1, \quad \xi_{i} \xi_{j}=-\xi_{j} \xi_{i} \quad(i \neq j) .
$$

It becomes a superalgebra under the parity $d\left(\xi_{i}\right)=1$. Let $\mathscr{C}_{n} \hat{\otimes} \mathscr{A}_{n}$ denote the super tensor product of the superalgebras $\mathscr{C}_{n}$ and $\mathscr{A}_{n}$. It is proved in 19 that the super tensor product $\mathscr{C}_{n} \hat{\otimes} \mathscr{A}_{n} \simeq \mathscr{B}_{n}$ as superalgebras under the map $\vartheta$ :

$$
\begin{aligned}
& \xi_{i} \otimes 1 \mapsto \tau_{i} \quad(1 \leq i \leq n), \\
& 1 \otimes \varsigma_{j} \mapsto \frac{1}{\sqrt{2}}\left(\tau_{j}-\tau_{j+1}\right) \omega_{j}, \quad(1 \leq j \leq n-1)
\end{aligned}
$$

where $\tau_{i}$ are defined above.

\section{Conjugacy classes of wreath products}

For a finite group $\Gamma$, set $\Gamma^{n}=\Gamma \times \cdots \times \Gamma$, the $n$-th direct product of $\Gamma$. The twisted hyperoctahedral group $\widetilde{H}_{n}$ acts on $\Gamma^{n}$ by permuting the factors:

$$
\begin{aligned}
z \cdot\left(g_{1}, \ldots, g_{n}\right) & =\left(g_{1}, \ldots, g_{n}\right), \\
a_{i} \cdot\left(g_{1}, \ldots, g_{n}\right) & =\left(g_{1}, \ldots, g_{n}\right), \quad i=1, \ldots, n \\
\sigma \cdot\left(g_{1}, \ldots, g_{n}\right) & =\left(g_{\sigma^{-1}(1)}, \ldots, g_{\sigma^{-1}(n)}\right), \sigma \in S_{n} .
\end{aligned}
$$


Let $\widetilde{H} \Gamma_{n}$ be the semi-direct product of $\Gamma^{n}$ by $\widetilde{H}_{n}$ with the multiplication:

$$
(g, a \sigma) \cdot\left(g^{\prime}, a^{\prime} \sigma^{\prime}\right)=\left(g \sigma\left(g^{\prime}\right), a \sigma\left(a^{\prime}\right) \sigma \sigma^{\prime}\right),
$$

where $g, g^{\prime} \in \Gamma^{n}, a, a^{\prime} \in \Pi_{n}, \sigma, \sigma^{\prime} \in S_{n}$. From now on, we write $H \Gamma_{n}$ for the wreath product of $S_{n}$ by $\Gamma \times \mathbb{Z}_{2}$. Note that $\widetilde{H} \Gamma_{n}$ is a double cover of $H \Gamma_{n}$. In the following we use the notations of [7] for $\widetilde{H} \Gamma_{n}$. For a subset $I=\left\{i_{1}, \ldots, i_{m}\right\}$ of $\{1, \ldots, n\}$, we set $a_{I}=a_{i_{1}} a_{i_{2}} \cdots a_{i_{m}} \in \widetilde{H}_{n}$ and $b_{I}=$ $b_{i_{1}} \cdots b_{i_{m}} \in H_{n}$, where $b_{i}(i=1, \ldots, n)$ are the generators of $\mathbb{Z}_{2}^{n}$.

We recall that a partition $\lambda$ of $n$ is a sequence of non increasing positive integers $\lambda_{i}$ called parts such that $\sum_{i} \lambda_{i}=n$. We denote by $l(\lambda)$ the number of parts or length of $\lambda$, and $\|\lambda\|=\lambda_{1}+\lambda_{2}+\cdots$ the weight of $\lambda$. A partition $\lambda$ is called a strict partition if all parts $\lambda_{i}$ are distinct. We also use the notation $\lambda=\left(1^{m_{1}(\lambda)} 2^{m_{2}(\lambda)} \ldots\right)$ for the partition $\lambda$, where $m_{k}(\lambda)$ is the multiplicity of $k$ in the parts $\lambda_{i}$ 's.

The conjugacy classes of $H \Gamma_{n}$ are parametrized by a pair of partitionvalued functions. For a cycle $\sigma=\left(i_{1} \ldots i_{m}\right) \in S_{n}$, the $\operatorname{supp}(\sigma)$ is defined to be $I=\left\{i_{1}, \ldots, i_{m}\right\}$. For $\left(g, b_{I} \sigma\right) \in H \Gamma_{n}$, the element $b_{I} \sigma$ can be uniquely written (up to order) as a product

$$
b_{I} \sigma=\left(b_{I_{1}} \sigma_{1}\right) \cdots\left(b_{I_{m}} \sigma_{m}\right)
$$

where $\sigma=\sigma_{1} \cdots \sigma_{m}$ is a product of disjoint cycles of $S_{n}$ and $I_{k} \subset \operatorname{supp}\left(\sigma_{k}\right)$ for $k=1, \ldots, m$ [7]. We call $b_{I_{a}} \sigma_{a}$ a signed cycle of $b_{I} \sigma$ with the sign $(-1)^{\left|I_{a}\right|}$. For each signed cycle $b_{I_{k}} \sigma_{k}$ with $\sigma_{k}=\left(j_{1}, \ldots, j_{m}\right)$ the signed cycle-product of $b_{I_{k}} \sigma_{k}$ is defined to be

$$
(-1)^{\left|I_{k}\right|} g_{j_{m}} g_{j_{m-1}} \cdots g_{j_{2}} g_{j_{1}} .
$$

For each $c \in \Gamma_{*}$, let $m_{k}^{+}(c)$ (resp. $\left.m_{k}^{-}(c)\right)(k \geq 0)$ be the number of $k$-cycles of the permutation $\sigma$ such that its positive (resp. negative) signed cycle-product lies in the conjugacy class $c$. For each $c \in \Gamma_{*}$, set

$$
\rho^{+}(c)=\left(1^{m_{1}^{+}(c)} 2^{m_{2}^{+}(c)} \ldots\right) \text { and } \rho^{-}(c)=\left(1^{m_{1}^{-}(c)} 2^{m_{2}^{-}(c)} \ldots\right),
$$

which are partitions indexed by $c \in \Gamma_{*}$. Then

$$
\rho^{+}=\left(\rho^{+}(c)\right)_{c \in \Gamma_{*}}, \quad \rho^{-}=\left(\rho^{-}(c)\right)_{c \in \Gamma_{*}}
$$

are two signed partition-valued functions on $\Gamma_{*}$. Moreover, $\rho=\left(\rho^{+}, \rho^{-}\right)$ defines a pair of partition-valued functions on $\Gamma_{*}$ with $l(\rho)=l\left(\rho^{+}\right)+l\left(\rho^{-}\right)$, where $l(\sigma)$ is the total length of $\sigma$, and $\left\|\rho^{+}\right\|+\left\|\rho^{-}\right\|=\|\rho\|$, the total weight of the partition-valued function. It is proved [7] that the conjugacy classes of $H \Gamma_{n}$ are indexed by pairs of partition-valued functions $\rho=\left(\rho^{+}, \rho^{-}\right)$such that $\|\rho\|=n$. In particular, $\rho=\left(\rho^{+}, \rho^{-}\right)$is called the type of the element $\left(g, b_{I} \sigma\right) \in H \Gamma_{n}$. Two elements of $H \Gamma_{n}$ are conjugate if and only if they have the same type.

We write $C_{\rho^{+}, \rho^{-}}$for the conjugacy class corresponding to elements of type $\left(\rho^{+}, \rho^{-}\right)$. Furthermore, as any element of $H \Gamma_{n}$ is of the form $\left(g, b_{I} \sigma\right)$, so a conjugacy class $C_{\rho^{+}, \rho^{-}}$is called even or odd if the cardinality $|I|$ is 
even or odd. Thus the general element of $\widetilde{H} \Gamma_{n}$ is of the form $\left(g,(-1)^{p} a_{I} \sigma\right)$ for $p= \pm 1$, where $(-1)^{p} a_{I} \sigma=(-1)^{p}\left(a_{I_{1}} \sigma_{1}\right) \cdots\left(a_{I_{q}} \sigma_{q}\right)$ and $\sigma=\sigma_{1} \cdots \sigma_{q}$ is a disjoint union of cycles in $S_{n}\left[7\right.$. We define the parity $p$ on $\widetilde{H} \Gamma_{n}$ by $p\left(g,(-1)^{p} a_{I} \sigma\right)=|I| \bmod 2$. Note that this parity generalizes the parity $p$ for $\widetilde{H}_{n}$ (below $(2.5)$ ). Therefore the group algebra $\mathbb{C}\left[\widetilde{H} \Gamma_{n}\right]$ becomes a superalgebra.

Let $\mathcal{P}_{n}\left(\Gamma_{*}\right)$ denote the set of partition-valued functions of $n$ on $\Gamma_{*}$. Let $\mathcal{O} \mathcal{P}_{n}\left(\Gamma_{*}\right)$ (resp. $\mathcal{S} \mathcal{P}_{n}\left(\Gamma_{*}\right)$ ) be the subset of $\mathcal{P}_{n}\left(\Gamma_{*}\right)$ such that each part is odd (resp. every partition is strict). For $i=0,1$, let $\mathcal{S P}_{n}^{i}\left(\Gamma_{*}\right)=\{\rho \in$ $\left.\mathcal{S} \mathcal{P}_{n}\left(\Gamma_{*}\right) \mid i=(n-l(\rho)) \bmod 2\right\}$. When $\Gamma=1$, the partition-valued functions become partitions, so we denote $\mathcal{O P}{ }_{n}, \mathcal{S} \mathcal{P}_{n}$ and $\mathcal{S P}_{n}^{i}(i=0,1)$. It is wellknown that the set $\mathcal{S P}{ }_{n}$ of strict partitions of $n$ is one-to-one correspondent to the set $\mathcal{O P} \mathcal{P}_{n}$ of partitions of $n$ by odd integers $\mathbf{1}$.

Denote by $D_{\rho^{+}, \rho^{-}}$the inverse image in $\widetilde{H} \Gamma_{n}$ of $H \Gamma_{n}$-conjugacy class $C_{\rho^{+}, \rho^{-}}$under the homomorphism $\theta_{n}$. For any $x \in C_{\rho^{+}, \rho^{-}}$, if $x$ is conjugate to $z x$ then $D_{\rho^{+}, \rho^{-}}$is also a $\widetilde{H} \Gamma_{n^{-}}$conjugacy class; Otherwise, $D_{\rho^{+}, \rho^{-}}$is a split class, in this case $C_{\rho^{+}, \rho^{-}}\left(\operatorname{resp} . D_{\rho^{+}, \rho^{-}}\right)$is called a split conjugacy class in $H \Gamma_{n}$ (resp. $\left.\widetilde{H} \Gamma_{n}\right)$. It is easy to see that only split conjugacy classes of $\widetilde{H} \Gamma_{n}$ can support nonzero spin character values.

Proposition 3.1. [7] The conjugacy class $C_{\rho^{+}, \rho^{-}}$in $H \Gamma_{n}$ is split into two conjugacy classes if and only if

(1) when $C_{\rho^{+}, \rho^{-}}$is even, we have $\rho^{+} \in \mathcal{O} \mathcal{P}_{n}\left(\Gamma_{*}\right)$ and $\rho^{-}=\emptyset$.

(2) when $C_{\rho^{+}, \rho^{-}}$is odd, we have $\rho^{+}=\emptyset$ and $\rho^{-} \in \mathcal{S P}_{n}^{1}\left(\Gamma_{*}\right)$.

In view of Prop. 3.1, when $C_{\rho^{+}, \rho^{-}}$is split, we can simply denote $D_{\rho}=$ $\theta_{n}^{-1}\left(C_{\rho^{+}, \rho^{-}}\right)=D_{\rho}^{+} \cup D_{\rho}^{-}$, where $D_{\rho}^{-}=z D_{\rho}^{+}$. Consequently the order of the centralizer of an element in $D_{\rho}$ of $\widetilde{H} \Gamma_{n}$ is given by

$$
\widetilde{Z}_{\rho}=2^{1+l(\rho)} Z_{\rho},
$$

where $Z_{\rho}=\prod_{c \in \Gamma_{*}, i \in \mathbb{N}} i^{m_{i}(c)} m_{i}(c) ! \zeta_{c}^{l(\rho(c))}$ and $\zeta_{c}$ is the order of the centralizer of an element in conjugacy class $c$ of $\Gamma$. For convenience, we also denote $z_{\rho(c)}=\prod_{i \in \mathbb{N}} i^{m_{i}(c)} m_{i}(c)$ !, so $Z_{\rho}=\prod_{c \in \Gamma_{*}} z_{\rho(c)}$.

\section{Character formulas for $\mathscr{A}_{n}$ and $\mathscr{B}_{n}$}

A spin representation of $\widetilde{S}_{n}$ (or $\left.\widetilde{H}_{n}\right)$ is an ordinary representation $\pi$ of $\widetilde{S}_{n}\left(\right.$ or $\left.\widetilde{H}_{n}\right)$ such that $\pi(z)=-1$. From superalgebra viewpoint Józefiak 10 computed the spin super-character values of $\widetilde{H}_{n}$ based on [17]. A general discussion was given earlier in Morris and Jones [14] on spin characters. The foundation of all these works is Schur's theory [17] that most character values of the spin symmetric group $\widetilde{S}_{n}$ are given by transition matrices between the basis of Schur's $Q$-functions $Q_{\lambda}$ and that of power-sum symmetric functions $p_{\mu}$ in the subring of symmetric functions generated by the power-sums of odd degrees: $p_{1}, p_{3}, \ldots$ For basic information on Schur's $Q$-functions, 
see Macdonald's classic monograph [11. As the exceptional spin character values are not given by symmetric functions, we will compute them for $\widetilde{H}_{n}$ in this section.

Recall that $\mathscr{A}_{n}$ a quotient of the group algebra $\mathbb{C}_{n}$ (see (2.7)). As a superalgebra $\mathscr{A}_{n}$ is a direct product of finitely many simple superalgebras. For background materials on superalgebras, we refer the reader to [8]. For a modern account of Schur's twisted tensor products, see also [18. Simple (complex) superalgebras have two types: (1) Type $M, M(r \mid s)$ is the block $2 \times 2$ matrices with the main diagonal blocks are $r \times r$-matrices and $s \times s$ matrices. The degree zero part consists of diagonal blocks and degree one is formed by off-diagonal blocks. (2) Type $Q, Q(n)$ is a block $2 \times 2$ matrices whose two main diagonals and off-diagonal are the same $n \times n$ matrices. The $\mathbb{Z}_{2}$-grading is similar to that of type $M$. We will call an irreducible supermodule type $M$ (resp. $Q$ ) if it corresponds to the simple superalgebra of type $M$ (resp. $Q$ ).

The Clifford superalgebra $\mathscr{C}_{n}$ is a simple superalgebra of type $M$ (resp. $Q$ ) according to $n$ even (resp. odd). Subsequently $L_{n}=\mathscr{C}_{n}$ is a simple $\mathscr{C}_{n}$-module of type $M$ (resp. $Q$ ) if $n$ is even (resp. odd).

Proposition 4.1. (Schur [17]) (1) The irreducible $\mathscr{A}_{n}$-supercharacters are indexed by $\mathcal{S P}_{n}$. For $\nu \in \mathcal{S P}_{n}$, let $\zeta_{\nu}$ be the character with type $\nu$, then it is completely determined by its values $\zeta_{\nu}^{\alpha}$ at the conjugacy class indexed by $\alpha \in \mathcal{O P}_{n}$ and

$$
Q_{\nu}=\sum_{\lambda \in \mathcal{O P}_{n}} 2^{\frac{l(\nu)+l(\lambda)+p(\nu)}{2}} z_{\lambda}^{-1} \zeta_{\nu}^{\lambda} p_{\lambda}
$$

where $p(\nu)$ is the parity of $\nu$ and $z_{\lambda}$ is the order of the centralizer of an element with cycle type $\lambda$ in $S_{n}$.

(2) The supercharacter $\zeta_{\nu}$ is of type $M$ (resp. Q) when $n-l(\nu)$ is even (resp. odd).

For $\nu \in \mathcal{S P}_{n}$, let $V_{\nu}$ be the $\mathscr{A}_{n}$-module associated with $\nu$. Since $\mathscr{B}_{n} \simeq$ $\mathscr{C}_{n} \hat{\otimes} \mathscr{A}_{n}$ and $\mathscr{C}_{n}$ has only one simple supermodule $L_{n}$, any irreducible super modules of $\mathscr{B}_{n}$ is a super tensor product $L_{n} \hat{\otimes} V_{\nu}$, where $V_{\mu}$ is irreducible $\mathscr{A}_{n^{-}}$ supermodule. Subsequently there is a one-to-one correspondence between irreducible $\mathscr{A}_{n}$-modules and irreducible $\mathscr{B}_{n}$-modules.

Proposition 4.2. (Józefiak [9]) (1) The irreducible supercharacters of $\mathscr{B}_{n}$ are completely indexed by the strict partitions of $n$. For $\nu \in \mathcal{S P}_{n}$, if $l(\nu)$ is even (resp. odd) then the corresponding supercharacter $\xi_{\nu}$ is of type $M$ (resp. Q).

(2) For each $\nu \in \mathcal{S P}_{n}$, the supercharacter values are determined by

$$
Q_{\nu}=\sum_{\lambda \in \mathcal{O P} \mathcal{P}_{n}} 2^{\left[\frac{l(\nu)}{2}\right]} z_{\lambda}^{-1} \xi_{\nu}^{\lambda} p_{\lambda} .
$$

where $[x]$ denotes the largest integer $\leq x$. 
Now we consider the relationship between characters of modules and super modules.

A spin representation $\pi$ of $\widetilde{S}_{n}$ (resp. $\widetilde{H}_{n}$ ) is called of double spin or self-associate spin if $\pi \cong(-1)^{s g n} \circ \pi$. Otherwise, $\pi$ and $\pi^{\prime}:=(-1)^{s g n} \circ \pi$ are called a pair of associate spin representations. Here $(-1)^{s g n}$ is the onedimensional sign representation of $\widetilde{S}_{n}$ (resp. $\widetilde{H}_{n}$ ). The following correspondence between ordinary spin characters and super spin characters is crucial for our discussion.

First of all, if $V$ is a super module of the superalgebra $\mathscr{A}_{n}$ (resp. $\mathscr{B}_{n}$ ), then $\chi_{V}(x)=\operatorname{trace}_{V}(x)=0$ for any element $x$ of degree one. On one hand, the character $\pi$ of a spin super module of type $M$ is also a double spin character by forgetting the super structure. If $\pi$ is the character of an irreducible supermodule of type $Q$, then $\pi=\chi^{+}+\chi^{-}$, where $\chi^{ \pm}$are a pair of irreducible associate spin characters corresponding to $\pi$. Here $\chi^{-}(x)=(-1)^{p(x)} \chi^{+}(x)$, where $p$ is the parity of the superalgebra. For convenience, we denote the difference $\nabla(\chi)=\chi^{+}-\chi^{-}$, which can be used to recover the associate spin characters from that of the supermodule. In summary, for $\widetilde{S}_{n}$ (or $\widetilde{H}_{n}$ ) we can get the ordinary spin character values on even conjugacy classes from the supercharacter formulas of $\mathscr{A}_{n}$ (or $\mathscr{B}_{n}$ ). Moreover, a double spin character is fully determined by its values on even conjugacy classes or degree 0 elements (as it vanishes on degree 1 elements), while associate spin characters may have non-zero values on odd conjugacy classes or degree 1 elements.

The following well-known result shows how associate spin characters take values at the odd conjugacy classes.

Proposition 4.3. (Schur [17]) For $\nu \in \mathcal{S P}_{n}^{1}$, there corresponds a pair of associate spin characters $\zeta_{\nu}^{+}$and $\zeta_{\nu}^{-}$of $\mathscr{A}_{n}$, and

$$
\zeta_{\nu}^{+}\left(\nu^{+}\right)=(\sqrt{-1})^{\frac{n-l(\nu)+1}{2}} \sqrt{\frac{\nu_{1} \nu_{2} \cdots \nu_{l}}{2}}, \quad \zeta_{\nu}^{+}\left(\mu^{+}\right)=0 \text { for } \mu \neq \nu \in \mathcal{S P}_{n}^{1} .
$$

Moreover $\zeta_{\nu}^{-}\left(\mu^{+}\right)=-\zeta_{\nu}^{+}\left(\mu^{+}\right)$, where $\mu^{+}$is an element in $D_{\mu}^{+}$.

On the other hand, if $V$ is a double spin irreducible $\mathscr{B}_{n}$-module, then $V$ can be naturally equipped with a $\mathscr{B}_{n}$-supermodule of type $\mathrm{M}$ as follows. Suppose $V \simeq V^{\prime}=(-1)^{s g n} \otimes V$ is irreducible, where $\operatorname{sgn}$ is the parity $p$ defined below (2.5). As a vector space $V^{\prime}=V$, the isomorphism map $H \in \mathrm{GL}(V)$ and satisfies that

$$
H g=(-1)^{s g(g)} g H, \quad \forall g \in \mathscr{B}_{n}
$$

Therefore $H^{2}$ commutes with $\mathscr{B}_{n}$, so it is a scalar by Schur's lemma. We can assume that $H^{2}=1$. Let $V_{0}$ be the 1-eigenspace of $H$ and $V_{1}$ the $(-1)$ eigenspace of $H$, then $V=V_{0} \oplus V_{1}$ gives rise to the desired $\mathscr{B}_{n}$-supermodule. Moreover, both $V_{i}$ are $\mathscr{B}_{n}^{(0)}$-modules affording the character $\chi_{V_{i}}$, then for 
$x \in \mathscr{B}_{n}^{(0)}$ one has that

$$
\begin{aligned}
\operatorname{tr}_{V}(x) & =\chi_{V}(x):=\chi_{V_{0}}(x)+\chi_{V_{1}}(x) \\
\operatorname{tr}_{V}(H x) & =\delta_{V}(x):=\chi_{V_{0}}(x)-\chi_{V_{1}}(x) .
\end{aligned}
$$

Here the latter is usually called the difference character of $V$ [17. We will extend this notation to the spin character of a super module of type $M$, when viewed as a character for the subalgebra of degree zero. Note that $\chi(x)=0$ if $x \in \mathscr{B}_{n}^{(1)}$ in this case.

Also, if $V$ is an irreducible associate spin $\mathscr{B}_{n}$-module, then $D(V)=V \oplus$ $V^{\prime}$ becomes an irreducible $\mathscr{B}_{n}$-supermodule where $D(V)_{0}=\{(v, v) \mid v \in V\}$, $D(V)_{1}=\{(v,-v) \mid v \in V\}$ and the action is induced from that of the ordinary module, i.e. $g^{(i)}(u, v)=\left(g^{(i)} u,(-1)^{i} g^{(i)} v\right)$ for $g^{(i)} \in \mathscr{B}_{n}^{(i)}$, the degree $i$ subspace of $\mathscr{B}_{n}^{(i)}$ (with respect to the parity $p$ ). Moreover, any irreducible associate spin module can be realized this way.

Conversely, any irreducible supermodule $U=U_{0} \oplus U_{1}$ of type $Q$ is of the form $D(V)$. In fact, let $(-1)^{p}:(x, y) \mapsto(x,-y)$ be the parity endomorphism of $U$. Then $V=\left\{\frac{1}{2}\left(v+(-1)^{p} v\right) \mid u \in U\right\}$ and $V^{\prime}=\left\{\frac{1}{2}\left(v-(-1)^{p} v\right) \mid u \in U\right\}$.

From now on till (4.7), we assume that $\left\{\mathscr{B}_{n}\right\}$ is a tower of finite-dimensional superalgebras:

$$
\mathscr{B}=\bigoplus_{n=0}^{\infty} \mathscr{B}_{n},
$$

where

$$
\mathbb{C}=\mathscr{B}_{0} \hookrightarrow \mathscr{B}_{1} \hookrightarrow \cdots \hookrightarrow \mathscr{B}_{n} \hookrightarrow \cdots
$$

and $\mathscr{B}$ a Hopf algebra under the natural multiplication and comultiplication:

$$
\begin{aligned}
& m: \mathscr{B}_{m} \hat{\otimes} \mathscr{B}_{n} \stackrel{\text { Ind }}{\longrightarrow} \mathscr{B}_{m+n}, \\
& \Delta: \mathscr{B}_{m} \longrightarrow \oplus_{i=0}^{m} \operatorname{Res} \mathscr{B}_{i} \hat{\otimes} \operatorname{Res} \mathscr{B}_{m-i} .
\end{aligned}
$$

Two nontrivial examples of the tower system $\left\{\mathscr{B}_{n}\right\}$ for superalgebras were studied first in [3, 7]. See also [2] for the case of $\widetilde{S}_{n}$.

For two spin supermodules $U$ and $V$ of $\mathscr{B}_{m}$ and $\mathscr{B}_{n}$, we define the super (outer)-tensor product $U \hat{\otimes} V$ as a $\mathscr{B}_{m} \hat{\otimes} \mathscr{B}_{n}$-supermodule by

$$
(x, y)(u \otimes v)=(-1)^{p(x) p(y)}(x u \otimes y v),
$$

where $x \in \mathscr{B}_{m}$ and $y \in \mathscr{B}_{n}$ are homogeneous elements. In particular, -1 of $\mathscr{B}_{m+n}$ is identified with $(-1,1)$ or $(1,-1)$ inside $\mathscr{B}_{m} \hat{\otimes} \mathscr{B}_{n} \hookrightarrow \mathscr{B}_{m+n}$. Then $U \hat{\otimes} V$ is a spin $\mathscr{B}_{m} \hat{\otimes} \mathscr{B}_{n}$-supermodule. Moreover, let $U$ and $V$ be irreducible supermodules for $\mathscr{B}_{m} \hat{\otimes} \mathscr{B}_{n}$ respectively, then we have that 8

(1) if both $U$ and $V$ are of type $M$, then $U \hat{\otimes} V$ is a simple $\mathscr{B}_{m} \hat{\otimes} \mathscr{B}_{n^{-}}$ supermodule of type $M$;

(2) if $U$ and $V$ are of different types, then $U \hat{\otimes} V$ is a simple $\mathscr{B}_{m} \hat{\otimes} \mathscr{B}_{n^{-}}$ supermodule of type $Q$; 
(3) if both $U$ and $V$ are of type $Q$, then $U \hat{\otimes} V \simeq N \oplus \bar{N}$ for some simple $\mathscr{B}_{m} \hat{\otimes} \mathscr{B}_{n}$-supermodules $N$ and $\bar{N}$ of type $M$.

The simple summands of $U \hat{\otimes} V$ in (3) are constructed as follows. Let $H_{1}$ (resp. $H_{2}$ ) be the endomorphism $(-1)^{p}$ for $U$ (resp. $V$ ). Then

$$
\begin{aligned}
& N_{0}=\left\{u \otimes v+H_{1} u \otimes H_{2} v+i\left(u \otimes H_{2} v+H_{1} u \otimes v\right) \mid u \in U, v \in V\right\}, \\
& N_{1}=\left\{u \otimes v-H_{1} u \otimes H_{2} v+i\left(u \otimes H_{2} v-H_{1} u \otimes v\right) \mid u \in U, v \in V\right\}, \\
& \bar{N}_{0}=\left\{u \otimes v+H_{1} u \otimes H_{2} v-i\left(u \otimes H_{2} v+H_{1} u \otimes v\right) \mid u \in U, v \in V\right\}, \\
& \bar{N}_{1}=\left\{u \otimes v-H_{1} u \otimes H_{2} v-i\left(u \otimes H_{2} v-H_{1} u \otimes v\right) \mid u \in U, v \in V\right\} .
\end{aligned}
$$

Let - be the map $N \rightarrow \bar{N}$ taking $i$ to $-i$ while keeping other part intact (like conjugation). Then $\alpha(x): x \mapsto(-1)^{p(x)} \bar{x}$ establishes a degree 1 isomorphism from $N$ to $\bar{N}$ as $\mathscr{B}_{m} \hat{\otimes} \mathscr{B}_{n}$-supermodules.

Using the method of [6] it can be verified that this super tensor product satisfies the associativity. Then we can pass this to usual spin modules as follows. Let $V$ be an irreducible spin module. If $V$ is an irreducible double spin module, then $V$ can be naturally given an irreducible supermodule structure of type $\mathrm{M}$ as described above (still denoted by the same symbol for the supermodule). If $V$ is an irreducible associate spin module, then $D(V)=V \oplus V^{\prime}$ is an irreducible supermodule of type $Q$, and $D(V)_{0} \simeq V$ as ordinary modules. The following useful result can be proved by a similar method to [8], and we have added new values for later consideration of multi-products.

Proposition 4.4. Let $\left\{\mathscr{B}_{n}\right\}$ be a tower system of finite dimensional superalgebras. Let $f$ and $g$ be the spin characters afforded by an irreducible $\mathscr{B}_{m}$-module $U$ and an irreducible $\mathscr{B}_{n}$-module $V$ respectively. Let $x=x_{0}+$ $x_{1} \in \mathscr{B}_{m}, y=y_{0}+y_{1} \in \mathscr{B}_{n}$.

(i) If both $U$ and $V$ are double spin, then the super tensor product $U \hat{\otimes} V$ is irreducible both as a supermodule of type $M$ and an ordinary double spin module for $\mathscr{B}_{m} \hat{\otimes} \mathscr{B}_{n}$. In this case, $f\left(x_{1}\right)=g\left(y_{1}\right)=f \circledast g\left(x_{i}, y_{1-i}\right)=0$ and

$$
\begin{aligned}
f \circledast g\left(x_{0}, y_{0}\right) & =f\left(x_{0}\right) g\left(y_{0}\right), \\
\delta(f \circledast g)\left(x_{i}, y_{i}\right) & =\delta(f)\left(x_{i}\right) \delta(g)\left(y_{i}\right) .
\end{aligned}
$$

(ii) If $U$ is double spin and $V$ is associate spin, then the super tensor product $U \hat{\otimes} D(V)$ is irreducible as a $\mathscr{B}_{m} \hat{\otimes} \mathscr{B}_{n}$-supermodule of type $Q$ and decomposes into $U \circledast V \oplus(U \circledast V)^{\prime}$ as an ordinary module, where $(U \circledast V)^{\prime}$ is the associated module of the irreducible module $U \circledast V$. In this case, $f\left(x_{1}\right)=f \circledast g\left(x_{1}, y_{0}\right)=0$ and the following identities hold.

$$
\begin{aligned}
f \circledast g\left(x_{0}, y_{0}\right) & =f\left(x_{0}\right) g\left(y_{0}\right), \\
f \circledast g\left(x_{0}, y_{1}\right) & =\delta(f)\left(x_{0}\right) g\left(y_{1}\right), \\
\Delta(f \circledast g)\left(x_{0}, y_{0}\right) & =f\left(x_{0}\right) \Delta(g)\left(y_{0}\right) .
\end{aligned}
$$

(iii) If both $U$ and $V$ are associate spin, so they give rise irreducible super spin modules $D(U)$ and $D(V)$ of type $Q$. The super tensor product 
$D(U) \hat{\otimes} D(V)$ decomposes into $W \oplus W$, where $W$ is an irreducible $\mathscr{B}_{m} \hat{\otimes} \mathscr{B}_{m}$ supermodule of type $M$. Denote by $W=U \circledast V$ when it is viewed as an ordinary irreducible module (up to isomorphism). In this case, we have that

$$
\begin{aligned}
f \circledast g\left(x_{1}, y_{0}\right) & =f \circledast g\left(x_{0}, y_{1}\right)=\delta(f \circledast g)\left(x_{0}, y_{0}\right)=0 \\
f \circledast g\left(x_{0}, y_{0}\right) & =2 f\left(x_{0}\right) g\left(y_{0}\right), \\
\delta(f \circledast g)\left(x_{1}, y_{1}\right) & =2 \sqrt{-1} f\left(x_{1}\right) g\left(y_{1}\right) .
\end{aligned}
$$

Let $\mu$ be a partition, and suppose $V_{i}(1 \leq i \leq m)$ is an irreducible spin (ordinary) $\mathscr{B}_{\mu_{i}}$-module. Since the starred tensor product $\circledast$ is associative [8, 6] (see our notation above), $V_{1} \circledast \cdots \circledast V_{s}$ is a well-defined spin $\hat{\otimes} \mathscr{B} \mu_{i}$ module. Let $f_{i}$ be the character of $V_{i}$, and assume that the first $r$ of them are irreducible double spin (corresponding to type $M$ ) and the latter $k=m-r$ are irreducible associate spin (corresponding to type $Q$ ). Then $f_{1} \circledast \cdots \circledast f_{m}$ is the character of an irreducible summand of the super tensor product

$$
V_{1} \hat{\otimes} \cdots \hat{\otimes} V_{r} \hat{\otimes} D\left(V_{r+1}\right) \hat{\otimes} \cdots \hat{\otimes} D\left(V_{r+k}\right) \text {. }
$$

Note that $V_{1} \circledast \cdots \circledast V_{m}$ is only defined up to isomorphism. Repeatedly using Prop. 4.4, we obtain that

$$
\begin{aligned}
& f_{1} \circledast \cdots \circledast f_{m}\left(x_{1}, \ldots, x_{m}\right)=2^{\left[\frac{k}{2}\right]} f_{1}\left(x_{1}\right) \cdots f_{k}\left(x_{m}\right), \\
& f_{1} \circledast \cdots \circledast f_{m}\left(x_{1}, \ldots x_{r}, y_{r+1}, \ldots, y_{m}\right) \\
& \quad=(2 \sqrt{-1})^{\frac{k-1}{2}} \delta\left(f_{1}\right)\left(x_{1}\right) \cdots \delta\left(f_{r}\right)\left(x_{r}\right) f_{r+1}\left(y_{r+1}\right) \cdots f_{m}\left(y_{m}\right), k \text { odd } \\
& f_{1} \circledast \cdots \circledast f_{m}\left(z_{1}, \ldots, z_{m}\right)=0, \quad \text { other type of } z_{i}{ }^{\prime} \mathrm{s}
\end{aligned}
$$

where $x_{i} \in \mathscr{B}_{\mu_{i}}^{(0)}, y_{i} \in \mathscr{B}_{\mu_{i}}^{(1)}, z_{i} \in \mathscr{B}_{\mu_{i}}$, and $[a]$ denotes the maximum integer $\leq a$. We remark the last formula (4.7) is verified with our updated trivial values stated in Prop. 4.4

Now we can compute the associated spin characters on odd conjugacy classes for $\widetilde{H}_{n}$. Let $r=\left[\frac{n}{2}\right]$, and $L_{n}=\mathscr{C}_{n} e$, where

$$
e=e_{1} e_{2} \cdots e_{r}, \quad e_{i}=\frac{1}{2}\left(1+\sqrt{-1} \xi_{2 i-1} \xi_{2 i}\right) .
$$

Then $e_{i}$ are commutative idempotents, and $L_{n}$ is a simple super $\mathscr{C}_{n}$-module.

Note that $\xi^{i}=\xi_{2 i-1} e_{i}=\frac{1}{2}\left(\xi_{2 i-1}+\sqrt{-1} \xi_{2 i}\right)(i=1, \ldots, r)$ generate an exterior algebra: $\left\{\xi^{i}, \xi^{j}\right\}=0$ due to the fact that $e_{i} \xi_{2 i-1} e_{i}=0$. For each $\varepsilon=$ $\left(\varepsilon_{1}, \ldots, \varepsilon_{r}\right) \in \mathbb{Z}_{2}^{r}$, define $\xi^{\varepsilon}=\xi_{1}^{\varepsilon_{1}} \xi_{3}^{\varepsilon_{2}} \cdots \xi_{2 r-1}^{\varepsilon_{r}} e=\left(\xi_{1}^{\varepsilon_{1}} e_{1}\right)\left(\xi_{3}^{\varepsilon_{2}} e_{2}\right) \cdots\left(\xi_{2 r-1}^{\varepsilon_{r}} e_{r}\right)$. If $n$ is even (resp. odd), then $\left\{\xi^{\varepsilon} \mid \varepsilon \in \mathbb{Z}_{2}^{r}\right\}$ (resp. $\left\{\xi^{\varepsilon} \mid \varepsilon \in \mathbb{Z}_{2}^{r}\right\} \cup\left\{\xi^{\varepsilon} \xi_{n} \mid \varepsilon \in\right.$ $\left.\left.\mathbb{Z}_{2}^{r}\right\}\right)$ is a basis of $L_{n}$. If $n$ is odd, define $c_{n} \in \operatorname{End}_{\mathscr{C}_{n}}^{1}\left(L_{n}\right)$ by $c_{n}\left(\xi^{\varepsilon} \xi_{n}^{\epsilon}\right)=$ $(-1)^{\sum_{i} \varepsilon_{i}+\epsilon}\left(\xi^{\varepsilon} \xi_{n}^{\epsilon+1}\right)$, where $\varepsilon=\left(\varepsilon_{1}, \ldots, \varepsilon_{r}\right) \in \mathbb{Z}_{2}^{r}$ and $\epsilon \in \mathbb{Z}_{2}$. Here $c_{n}^{2}=-1$.

By our earlier discussion, irreducible $\widetilde{H}_{n}$-supermodules are indexed by strict partitions $\nu$ and they are type $M$ (resp. $Q$ ) iff $l(\nu)$ are even (resp. odd). Correspondingly double irreducible spin characters are indexed by strict partitions $\nu$ with even $l(\nu)$, and pairs of irreducible associate spin characters are indexed by strict partitions $\nu$ with odd $l(\nu)$. 
Let $\xi_{\nu}$ be the character of the irreducible supermodule associated with $\nu$. If $l(\nu)$ is even, then $\xi_{\nu}$ vanishes at split conjugacy classes of degree one, i.e., $\xi_{\nu}\left(D_{\rho}^{ \pm}\right)=0$ for any odd strict partition $\rho \in \mathcal{S P}^{1}$ (as $D_{\rho}^{ \pm}$are the only split odd classes by Theorem 3.1). Therefore, $\xi_{\nu}$ 's are completely given by Schur's $Q$-functions in this case (see Prop. 4.2). So we are left with the task of fixing $\xi_{\nu}$ with odd $l(\nu)$. In this case $\xi_{\nu}$ breaks into a pair of associate spin characters $\xi_{\nu}^{ \pm}$. Again the character values on the even split classes are given by Schur's $Q$-functions, and we only need to worry about $\xi_{\nu}$ on the odd split conjugacy classes $\mathcal{S} \mathcal{P}^{1}$. The following result computes the remaining character values for $\mathscr{B}_{n}$ that correspond to strict partitions of odd lengths.

TheOREM 4.5. For $\nu=\left(\nu_{1}, \ldots, \nu_{l}\right) \in \mathcal{S P}_{n}$, let $\xi_{\nu}$ be the corresponding spin supercharacter of $\mathscr{B}_{n}$. When $l(\nu)$ is odd, $\xi_{\nu}$ is decomposed into two associate ordinary spin characters $\xi_{\nu}^{ \pm}$of $\widetilde{H}_{n}$. Moreover, $\left(\xi_{\nu}^{ \pm}\right)\left(\mu^{+}\right)=0$ for $\mu \neq \nu \in \mathcal{S P}_{n}^{1}$ and for $\mu=\nu$,

$$
\xi_{\nu}^{ \pm}\left(\nu^{+}\right)= \pm 2^{\frac{l(\nu)}{2}} \cdot(\sqrt{-1})^{\frac{n-m}{2}} \sqrt{\frac{\nu_{1} \nu_{2} \cdots \nu_{l(\nu)}}{2}},
$$

where $\mu^{+} \in D_{\mu}^{+}=\theta^{-1}\left(C_{\emptyset, \mu}\right)$ and $m$ is the number of odd parts in $\nu$.

Proof. We prove the formula by induction on $l(\nu)$. We first consider the case $\nu=(n)$, and divide into two cases according to the parity of $n$.

Case (1). If $n$ is odd, the $\mathscr{C}_{n}$-module $L_{n}$ is of type $Q$ and the $\mathscr{A}_{n^{-}}$ character $\zeta_{(n)}$ is of type $M$. This is not a special case of the formula, but we need to compute the difference character for the inductive procedure. The element $\sigma^{((n), \emptyset)}$ of $\mathscr{B}_{n}$ is given by $\sigma^{((n), \emptyset)}=\omega_{1} \omega_{2} \cdots \omega_{n-1} \in \mathscr{B}_{n}^{(0)}$. Using Eq. (2.10), we obtain that

$$
\vartheta^{-1}\left(\omega_{1} \omega_{2} \cdots \omega_{n-1}\right)=\left(\frac{1}{\sqrt{2}}\left(\xi_{1}-\xi_{2}\right) \otimes \varsigma_{1}\right)\left(\frac{1}{\sqrt{2}}\left(\xi_{2}-\xi_{3}\right) \otimes \varsigma_{2}\right) \cdots,
$$

From [19], we have $\varsigma_{i} \xi_{j}=-\xi_{j} \varsigma_{i}$, then rearrange the above product into the following form

$$
2^{-\frac{n-1}{2}}(-1)^{\frac{(n-1)(n-2)}{2}} \prod_{j=1}^{n-1}\left(\xi_{j}-\xi_{j+1}\right) \otimes\left(\varsigma_{1} \varsigma_{2} \cdots \varsigma_{n-1}\right) .
$$

Therefore, the value of $\xi_{(n)}^{+}=\frac{1}{2} \Delta\left(\operatorname{ch}\left(L_{n}\right) \hat{\otimes} \zeta_{(n)}\right)$ at the even element equals to

$$
\begin{aligned}
& \frac{1}{2} \operatorname{ch}\left(L_{n}\right) \hat{\otimes} \zeta_{(n)}\left(\vartheta^{-1}\left(\sigma^{((n), \emptyset)}\right)\right) \\
= & (-1)^{\frac{(n-1)(n-2)}{2}} 2^{-\frac{n+1}{2}} \operatorname{ch}\left(L_{n}\right)\left(\prod_{j=1}^{n-1}\left(\xi_{j}-\xi_{j+1}\right)\right) \zeta_{(n)}\left(\varsigma^{(n)}\right),
\end{aligned}
$$


where $\varsigma^{(n)}=\varsigma_{1} \varsigma_{2} \cdots \varsigma_{n-1}$. As the coefficient of 1 in $\prod_{j=1}^{n-1}\left(\xi_{j}-\xi_{j+1}\right)$ is $(-1)^{(n-1) / 2}$, it follows that

$$
\operatorname{ch}\left(L_{n}\right)\left(\prod_{j=1}^{n-1}\left(\xi_{j}-\xi_{j+1}\right)\right)=2^{\frac{n+1}{2}} \cdot(-1)^{\frac{n-1}{2}} .
$$

On the other hand, $\delta\left(\zeta_{(n)}\right)\left(\varsigma^{(n)}\right)=(\sqrt{-1})^{(n-1) / 2} \sqrt{n}$ (note $n$ is odd). So we get the difference of the character is equal to

$$
\begin{aligned}
\delta\left(\xi_{(n)}^{+}\right)((n)) & =\frac{1}{2} \operatorname{ch}\left(L_{n}\right) \hat{\otimes} \delta\left(\zeta_{(n)}\right)\left(\vartheta^{-1}\left(\sigma^{((n), \emptyset)}\right)\right. \\
& =(\sqrt{-1})^{\frac{n-1}{2}} \sqrt{n}
\end{aligned}
$$

Case (2). When $n$ is even, $\sigma^{(\emptyset,(n))}=\omega_{0} \omega_{1} \cdots \omega_{n-1} \in \mathscr{B}_{n}^{(1)}$ and its preimage equals to

$$
\begin{aligned}
& \vartheta^{-1}\left(\omega_{0} \omega_{1} \cdots \omega_{n-1}\right) \\
= & 2^{-\frac{n-1}{2}} \cdot(-1)^{\frac{(n-1)(n-2)}{2}} \xi_{1} \prod_{j=1}^{n-1}\left(\xi_{j}-\xi_{j+1}\right) \otimes\left(\varsigma_{1} \varsigma_{2} \cdots \varsigma_{n-1}\right),
\end{aligned}
$$

which is a product of an even element and an odd element. Note that $\operatorname{ch}\left(L_{n}\right)\left(\xi_{1} \prod_{j=1}^{n-1}\left(\xi_{j}-\xi_{j+1}\right)\right)=2^{\frac{n}{2}} \cdot(-1)^{\frac{n-2}{2}}$ and it is of type $M$ due to $n$ being even. Since $\xi_{(n)}$ is of type $Q$, so $\xi_{(n)}^{+}=\frac{1}{2} \Delta\left(\xi_{(n)}\right)$. It follows from $\zeta_{(n)}^{+}(n)=(\sqrt{-1})^{\frac{n}{2}} \sqrt{\frac{n}{2}}$ (Prop. 4.3) that

$$
\begin{aligned}
& \xi_{(n)}^{+}((n))=\frac{1}{2} \Delta\left(\operatorname{ch}\left(L_{n}\right) \hat{\otimes} \zeta_{(n)}^{+}\right)\left[\vartheta^{-1}\left(\sigma^{(\emptyset,(n))}\right)\right] \\
= & 2^{-\frac{n-1}{2}} \cdot(-1)^{\frac{(n-1)(n-2)}{2}} \operatorname{ch}\left(L_{n}\right)\left(\xi_{1} \prod_{j=1}^{n-1}\left(\xi_{j}-\xi_{j+1}\right)\right) \cdot \zeta_{(n)}^{+}\left(\varsigma_{(n)}\right) \\
= & 2^{-\frac{n-1}{2}} \cdot(-1)^{\frac{(n-1)(n-2)}{2}} \cdot(-1)^{\frac{n-2}{2}} 2^{\frac{n}{2}} \cdot(\sqrt{-1})^{\frac{n}{2}} \sqrt{\frac{n}{2}} \\
= & (\sqrt{-1})^{\frac{n}{2}} \sqrt{n} .
\end{aligned}
$$

Next, suppose $\nu=\left(\nu_{1}, \nu_{2}, \ldots, \nu_{l}\right)$ is any strict partition of $n$ with odd $l=$ $l(\nu)$, say $\nu_{1}, \ldots, \nu_{m}$ are odd and $\nu_{m+1}, \ldots, \nu_{l}$ are even, then by (4.5) [4.6) it follows that

$$
\begin{aligned}
\xi_{\nu}^{+}\left(\nu^{+}\right)= & 2^{\left[\frac{l}{2}\right]}(\sqrt{-1})^{\frac{\nu_{1}+\cdots+\nu_{m}-m}{2}} \cdot \sqrt{\nu_{1} \cdots \nu_{m}} \\
& \cdot(\sqrt{-1})^{\frac{\nu_{m+1}+\cdots+\nu_{l}}{2}} \cdot \sqrt{\nu_{m+1} \cdots \nu_{l}} \\
= & 2^{\frac{l}{2}}(\sqrt{-1})^{\frac{n-m}{2}} \sqrt{\frac{\nu_{1} \nu_{2} \cdots \nu_{l}}{2}} .
\end{aligned}
$$


Note that $\left\langle\xi_{\nu}^{+}, \xi_{\nu}^{+}\right\rangle_{\mathcal{S P}}{ }_{n}^{1}=1 / 2$. On the other hand

$$
\begin{aligned}
\left\langle\xi_{\nu}^{+}, \xi_{\nu}^{+}\right\rangle_{\mathcal{S P}}^{1} & \geq \frac{1}{\left|\widetilde{H}_{n}\right|} \sum_{g \in D_{\emptyset, \nu}^{+} \cup D_{\emptyset, \nu}^{-}}\left|\xi_{\nu}^{+}(g)\right|^{2} \\
& =\frac{1}{2^{1+l} z_{\nu}} 2 \cdot\left|\xi_{\nu}^{+}\left(\nu^{+}\right)\right|^{2} \\
& =\frac{1}{2^{1+l} \nu_{1} \cdots \nu_{l}} \cdot 2 \cdot\left|2^{\frac{l}{2}} \sqrt{\frac{\nu_{1} \cdots \nu_{l}}{2}}\right|^{2}=\frac{1}{2}
\end{aligned}
$$

Therefore $\xi_{\nu}^{+}\left(\mu^{+}\right)=0$ for $\mu \neq \nu$.

For $\nu \in \mathcal{S P}_{n}$, we have that $\xi_{\nu}^{+}(\alpha)=2^{\frac{l\left(\alpha^{+}\right)-1}{2}} \zeta_{\nu}^{+}\left(\alpha^{+}\right)$for $\alpha \in \mathcal{O P} \mathcal{P}_{n}$ and $\xi_{\nu}^{+}\left(\nu^{+}\right)=2^{\frac{l(\nu)}{2}}(\sqrt{-1})^{\frac{l(\nu)-m-1}{2}} \zeta_{\nu}^{+}\left(\nu^{+}\right)$for $\nu \in \mathcal{S P}_{n}^{1}$, where $\alpha^{+} \in D_{\alpha}^{+}$and $m$ is the number of odd parts in $\nu$.

\section{Spin characters of $\widetilde{H} \Gamma_{n}$.}

We first recall the parametrization of irreducible spin $\widetilde{H} \Gamma_{n}$-supermodules.

Proposition 5.1. [7] The irreducible double spin representations over $\widetilde{H} \Gamma_{n}$ are indexed by strict partition valued functions with even length on $\Gamma^{*}$, and the pairs of irreducible associate spin representations are indexed by strict partition-valued functions with odd length on $\Gamma^{*}$.

A spin class function on $\widetilde{H} \Gamma_{n}$ is a function $f: \widetilde{H} \Gamma_{n} \longrightarrow \mathbb{C}$ such that $f(z x)=-f(x)$ for any element $x$ of a conjugacy class. Let $R\left(\widetilde{H} \Gamma_{n}\right)$ be the space of complex-valued spin class functions on $\widetilde{H} \Gamma_{n}$. The usual bilinear form is defined by

$$
\begin{aligned}
\langle f, g\rangle_{\widetilde{H} \Gamma_{n}} & =\frac{1}{\left|\widetilde{H} \Gamma_{n}\right|} \sum_{\tilde{x} \in \widetilde{H} \Gamma_{n}} f(\tilde{x}) g\left(\tilde{x}^{-1}\right) \\
& =\sum_{\rho \in \mathcal{O} \mathcal{P}_{n}\left(\Gamma_{*}\right) \cup \mathcal{S} \mathcal{P}_{n}^{1}\left(\Gamma_{*}\right)}\left[\frac{1}{\left|\widetilde{H} \Gamma_{n}\right|} \sum_{\widetilde{x} \in D_{\rho}^{+} \cup D_{\rho}^{-}} f(\widetilde{x}) \overline{g(\widetilde{x})}\right] \\
& =\sum_{\rho \in \mathcal{O} \mathcal{P}_{n}\left(\Gamma_{*}\right) \cup \mathcal{S} \mathcal{P}_{n}^{1}\left(\Gamma_{*}\right)} \frac{1}{2^{l(\rho)} Z_{\rho}} f\left(D_{\rho}^{+}\right) \overline{g\left(D_{\rho}^{+}\right)}
\end{aligned}
$$

where and $f, g \in R\left(\widetilde{H} \Gamma_{n}\right)$.

A spin super class function $\phi$ on $\widetilde{H} \Gamma_{n}$ is a spin class function such that it vanishes further on odd strict conjugacy classes. Let $R^{-}\left(\widetilde{H} \Gamma_{n}\right)$ be the $\mathbb{C}$-span space of spin super class functions on $\widetilde{H} \Gamma_{n}$. It is easy to see that the spin super-characters of $\widetilde{H} \Gamma_{n}$ form a $\mathbb{C}$-basis of $R^{-}\left(\widetilde{H} \Gamma_{n}\right)$, therefore the 
standard bilinear form on $R^{-}\left(\widetilde{H} \Gamma_{n}\right)$ is given by

$$
\begin{array}{r}
\langle\phi, \varphi\rangle_{\widetilde{H} \Gamma_{n}}=\frac{1}{\left|\widetilde{H} \Gamma_{n}\right|} \sum_{\tilde{x} \in \widetilde{H} \Gamma_{n}} \phi(\tilde{x}) \varphi\left(\tilde{x}^{-1}\right) \\
=\sum_{\rho \in \mathcal{O}_{n}\left(\Gamma_{*}\right)} \frac{1}{2^{l(\rho)} Z_{\rho}} \phi\left(D_{\rho}^{+}\right) \overline{\varphi\left(D_{\rho}^{+}\right)},
\end{array}
$$

where $\phi, \varphi \in R^{-}\left(\widetilde{H} \Gamma_{n}\right)$, as the (super)character values vanish on odd split conjugacy classes.

Wang and the second author [7] used the vertex operator calculus to compute spin characters of all simple $\widetilde{H} \Gamma_{n}$-supermodules. In terms of our current discussion this means that the ordinary irreducible spin character values of $\widetilde{H} \Gamma_{n}$ at even conjugacy classes are determined by transition matrix between generalized (or more generally wreath product) Schur $Q$-functions and power-sum symmetric functions. Moreover, if $\chi$ is an irreducible spin character of $\widetilde{H} \Gamma_{n}$ then

$$
\begin{gathered}
\langle\chi, \chi\rangle_{\mathcal{O P}}\left(\Gamma_{*}\right)=1,\langle\chi, \chi\rangle_{\mathcal{S P}_{n}^{1}\left(\Gamma_{*}\right)}=0 \text { for } \chi \text { a double spin, } \\
\langle\chi, \chi\rangle_{\mathcal{O P}_{n}\left(\Gamma_{*}\right)}=\langle\chi, \chi\rangle_{\mathcal{S P}_{n}^{1}\left(\Gamma_{*}\right)}=\frac{1}{2} \text { for } \chi \text { an associate spin. }
\end{gathered}
$$

In the following we will compute the values of the associate spin characters at odd strict conjugacy classes, which are not given by the theory of symmetric functions or vertex operator calculus (see table 1). Recall that the general element $\left(g, \pm a_{I} \sigma\right) \in \widetilde{H} \Gamma_{n}$ is even or odd according to the parity of the cardinality of $I$.

Let $U_{\gamma}$ be the irreducible $\Gamma$-module afforded by the irreducible character $\gamma \in \Gamma^{*}$. For each strict partition $\nu$ of $n$ let $V_{\nu}$ be the corresponding irreducible spin $\widetilde{H}_{n}$-supermodule afforded by the spin super character $\xi_{\nu}$. Now let $\lambda=\left(\lambda_{\gamma}\right)_{\gamma \in \Gamma^{*}}$ to be a partition-valued function on $\Gamma^{*}$, we recall the following result which describes the corresponding simple supermodule of $\widetilde{H} \Gamma_{\lambda}$.

Proposition 5.2. [7] For each strict partition-valued function $\lambda=$ $\left(\lambda_{\gamma}\right)_{\gamma \in \Gamma^{*}} \in \mathcal{S P}_{n}\left(\Gamma^{*}\right)$, the tensor product $\hat{\bigotimes}_{\gamma \in \Gamma^{*}}\left(U_{\gamma}^{\otimes\left|\lambda_{\gamma}\right|} \otimes V_{\lambda_{\gamma}}\right)$ decomposes completely into $2^{\left[\frac{m}{2}\right]}$ copies of an irreducible spin $\widetilde{H} \Gamma_{\lambda}$-supermodule $W_{\lambda}$, where $m$ denotes the number of the partitions $\lambda_{\gamma}$ such that $l\left(\lambda_{\gamma}\right)$ is odd. Then the induced supermodule Ind $\underset{\widetilde{H} \Gamma_{\lambda}}{\widetilde{H} \Gamma_{n}} W_{\lambda}$ is the irreducible spin $\widetilde{H} \Gamma_{n}$-supermodule indexed by $\lambda$, and it is of type $M$ or $Q$ according to $l(\lambda)$ is even or odd.

Let $\lambda$ be a partition-valued function $\lambda=\left(\lambda_{x}\right)_{x \in X}$, where

$$
\lambda_{x}=\left(\lambda_{x}(1), \lambda_{x}(2), \ldots\right)
$$

is a partition for each color $x \in X$. We denote by $\bar{\lambda}$ the ordinary partition obtained from $\lambda$ by forgetting all the colors, i.e. its parts consist of all $\lambda_{x}(i), x \in X$. For a finite set $X$, let $\lambda=\left(\lambda_{x}\right)_{x \in X} \in \mathcal{S P}_{n}(X)$ and define 
$J_{\lambda}=\left\{x \in X \mid l\left(\lambda_{x}\right)\right.$ is odd $\}$ and $J_{\lambda}^{\prime}=\left\{x \in X \mid l\left(\lambda_{x}\right)\right.$ is even $\}$ such that $J_{\lambda} \cup J_{\lambda}^{\prime}=X$. We denote $l\left(J_{\lambda}\right)=\sum_{x \in J_{\lambda}} l\left(\lambda_{x}\right),\|\lambda\|_{J_{\lambda}}=\sum_{x \in J_{\lambda}}\left|\lambda_{x}\right|$ and $m_{J_{\lambda}}=\sum_{x \in J_{\lambda}} m_{\lambda_{x}}$, where $m_{\lambda_{x}}$ is the number of odd parts in $\lambda_{x}$. For each strict partition $\lambda_{\gamma}$ let $\xi_{\lambda_{\gamma}}$ be the corresponding irreducible spin supercharacter of $\widetilde{H}_{\left|\lambda_{\gamma}\right|}$.

It follows from Proposition 5.2 that the irreducible super-character

$$
\operatorname{ch}\left(W_{\lambda}\right)=2^{-\left[\frac{\left|J_{\lambda}\right|}{2}\right]} \prod_{\gamma \in \Gamma^{*}}\left(\gamma^{\otimes\left|\lambda_{\gamma}\right|} \otimes \xi_{\lambda_{\gamma}}\right) .
$$

The induced character $\chi_{\lambda}=\operatorname{Ind} \underset{\widetilde{H} \Gamma_{\lambda}}{\widetilde{H} \Gamma_{n}} \operatorname{ch}\left(W_{\lambda}\right)$ is a double spin character when $l(\lambda)$ is even and $\chi_{\lambda}^{ \pm}=\operatorname{Ind} \underset{\widetilde{H} \Gamma_{\lambda}}{\widetilde{H} \Gamma_{n}} \operatorname{ch}\left(W_{\lambda}^{ \pm}\right)$are associate spin characters when $l(\lambda)$ is odd.

For a partition-valued function $\left(\lambda_{\gamma}\right)=\left(\lambda_{1}, \ldots, \lambda_{l}\right)_{\gamma}$, the partition $\overline{\left(\lambda_{\gamma}\right)}=$ $\left(\lambda_{1}, \ldots, \lambda_{l}\right)$ gives rise to a collection $[\gamma]$ of partition-valued functions $\rho=$ $\left(\rho_{c}\right)_{c \in \Gamma_{*}}=\left(\rho_{c_{1}}, \ldots, \rho_{c_{\left|\Gamma_{*}\right|}}\right)$ on $\Gamma_{*}$ by $\bar{\rho}=\left(\bar{\rho}_{\gamma_{1}}, \ldots, \bar{\rho}_{\gamma_{\left|\Gamma^{*}\right|}}\right)$ such that $\bar{\rho}=\overline{\left(\lambda_{\gamma}\right)}$. Clearly $|[\gamma]|=\left|\Gamma_{*}\right|^{l(\rho)}$. For brevity a partition-valued function inside $[\gamma]$ is usually written as $\rho_{\gamma}$. Now suppose $\rho=\left(\rho_{c}\right)_{c \in \Gamma_{*}} \in \mathcal{S P}_{n}^{1}\left(\Gamma_{*}\right)$ is an odd strict partition-valued function and $\lambda_{\gamma} \in \mathcal{S} \mathcal{P}_{\left|\lambda_{\gamma}\right|}\left(\Gamma^{*}\right)$ with $l(\lambda)$ being odd. Then using (4.6) we have

$$
\begin{aligned}
\operatorname{ch}\left(W_{\lambda}^{+}\right)\left(D_{\rho}^{+}\right)= & (2 \sqrt{-1})^{\left[\frac{\left|J_{\lambda}\right|}{2}\right]} \prod_{\gamma \in J_{\lambda}} \gamma^{\otimes\left|\lambda_{\gamma}\right|} \otimes \xi_{\lambda_{\gamma}}^{+}\left(D_{\rho_{\gamma}}^{+}\right) . \\
& \prod_{\gamma \in J_{\lambda}^{\prime}} \delta\left(\gamma^{\otimes\left|\lambda_{\gamma}\right|} \otimes \xi_{\lambda_{\gamma}}\right)\left(D_{\rho_{\gamma}}^{+}\right) .
\end{aligned}
$$

By Prop. [5.1, irreducible $\widetilde{H} \Gamma_{n}$-supermodules are indexed by strict partition-valued functions $\lambda \in \mathcal{S P}\left(\Gamma^{*}\right)$ and they are type $M$ (resp. $Q$ ) iff $l(\lambda)$ are even (resp. odd). Correspondingly double irreducible spin characters are indexed by strict partition values functions $\lambda$ with even $l(\lambda)$, and pairs of irreducible associate spin characters are indexed by strict partitions $\lambda \in$ $\mathcal{S P}\left(\Gamma^{*}\right)$ with odd $l(\lambda)$.

Let $\xi_{\lambda}$ be the character of the irreducible supermodule associated with partition-valued function $\lambda$. If $l(\nu)$ is even, then $\xi_{\lambda}$ vanishes at split conjugacy classes of degree one, i.e., $\xi_{\lambda}\left(D_{\rho}^{ \pm}\right)=0$ for any odd strict partitionvalued function $\rho \in \mathcal{S P}^{1}\left(\Gamma_{*}\right)$ (see Proposition 3.1). Therefore, $\xi_{\lambda}$ 's are completely given by the vertex operator calculus in this case (see [7]). So we are left with the task of fixing $\xi_{\lambda}$ with odd $l(\lambda)$. In this case $\xi_{\lambda}$ breaks into a pair of associate spin characters $\xi_{\lambda}^{ \pm}$. Again the character values on the even split classes are given by vertex operator calculus, and we only need to worry about $\xi_{\lambda}$ on the odd split conjugacy classes $D_{\rho}, \rho \in \mathcal{S P}^{1}\left(\Gamma_{*}\right)$. The following result computes the remaining character values for $\widetilde{H} \Gamma_{n}$ that correspond to strict partition-valued functions of odd lengths. 
The following theorem gives the irreducible associate spin character values at conjugacy classes indexed by odd strict partition-valued functions for $\widetilde{H} \Gamma_{n}$.

THEOREM 5.3. Let $\chi_{\lambda}^{+}$be an associate spin character (i.e. $\lambda=\left(\lambda_{\gamma}\right)_{\gamma \in \Gamma^{*}}$ $\in \mathcal{S P}_{n}\left(\Gamma^{*}\right), l(\lambda)$ odd $)$, for $\rho \in \mathcal{S P}_{n}^{1}\left(\Gamma_{*}\right)$ we have

(i) when $\rho=\left(\rho_{\gamma}\right)_{\gamma \in \Gamma^{*}}$ such that $\rho_{\gamma} \in \mathcal{O S P P}_{\left|\rho_{\gamma}\right|}\left(\Gamma_{*}\right)$ for $\gamma \in J_{\lambda}^{\prime}$ and $\rho_{\gamma} \in\left[\lambda_{\gamma}\right]$ for $\gamma \in J_{\lambda}$ then

$$
\begin{aligned}
\chi_{\lambda}^{+}\left(D_{\rho}^{ \pm}\right)= & \pm \prod_{\gamma \in \Gamma^{*}}\left(\prod_{c \in \Gamma_{*}} \gamma(c)^{l\left(\rho_{\gamma}(c)\right)}\right) \cdot \prod_{\gamma \in J_{\lambda}^{\prime}} \xi_{\lambda_{\gamma}}\left(t_{\rho_{\gamma}}\right) . \\
& 2^{\frac{l\left(J_{\lambda}\right)}{2}}(\sqrt{-1}) \frac{\|\rho\|_{J_{\lambda}-m_{J_{\lambda}}}}{2} \sqrt{\frac{\prod_{\gamma \in J_{\lambda}} \prod_{c \in \Gamma_{*}} z_{\rho_{\gamma}(c)}}{2}},
\end{aligned}
$$

where $t_{\rho_{\gamma}} \in D_{\rho_{\gamma}}^{+}$and the value $\prod_{\gamma \in J_{\lambda}^{\prime}} \xi_{\lambda_{\gamma}}\left(t_{\rho_{\gamma}}\right)$ is given by Schur Q-functions.

(ii) $\chi_{\lambda}^{+}\left(D_{\rho}^{ \pm}\right)=0$, otherwise.

Proof. (i) We have already seen that

$$
\begin{gathered}
\delta\left(\gamma^{\otimes\left|\lambda_{\gamma}\right|} \otimes \xi_{\lambda_{\gamma}}\right)\left(D_{\rho_{\gamma}}^{ \pm}\right)= \pm \prod_{c \in \Gamma_{*}} \gamma(c)^{l\left(\rho_{\gamma}(c)\right)} \cdot \delta\left(\xi_{\lambda_{\gamma}}\right)\left(t_{\rho_{\gamma}}\right) ; \\
\gamma^{\otimes\left|\lambda_{\gamma}\right|} \otimes \xi_{\lambda_{\gamma}}^{+}\left(D_{\rho_{\gamma}}^{ \pm}\right)= \pm \prod_{c \in \Gamma_{*}} \gamma(c)^{l\left(\rho_{\gamma}(c)\right)} \cdot \xi_{\lambda_{\gamma}}^{+}\left(t_{\rho_{\gamma}}\right) .
\end{gathered}
$$

Note that we will show that there is only one left coset $T$ of $\widetilde{H} \Gamma_{\lambda}$ in $\widetilde{H} \Gamma_{n}$ such that $\left(g, a_{I} s\right) T=T$. Suppose there are $K_{\rho}$ such cosets. It follows from Prop. 5.2 that (as $l(\lambda)$ is odd, so $\left|J_{\lambda}\right|$ is odd)

$$
\begin{aligned}
\chi_{\lambda}^{+}\left(D_{\rho}^{ \pm}\right)= & \pm K_{\rho} \cdot 2^{\frac{\left|J_{\lambda}\right|-1}{2}} \prod_{\gamma \in \Gamma^{*}}\left(\prod_{c \in \Gamma_{*}} \gamma(c)^{l\left(\rho_{\gamma}(c)\right)}\right) \cdot \prod_{\gamma \in J_{\lambda}^{\prime}} \xi_{\lambda_{\gamma}}\left(t_{\rho_{\gamma}}\right) . \\
& \prod_{\gamma \in J_{\lambda}}\left(2^{\frac{l\left(\lambda_{\gamma}\right)}{2}}(\sqrt{-1})^{\frac{\left|\rho_{\gamma}\right|-m_{\lambda}}{2}} \sqrt{\left.\frac{\prod_{c \in \Gamma_{*}} z_{\rho_{\gamma}(c)}}{2}\right)}\right. \\
= & \pm K_{\rho} \prod_{\gamma \in \Gamma^{*}}\left(\prod_{c \in \Gamma_{*}} \gamma(c)^{l\left(\rho_{\gamma}(c)\right)}\right) \cdot \prod_{\gamma \in J_{\lambda}^{\prime}} \xi_{\lambda_{\gamma}}\left(t_{\rho_{\gamma}}\right) . \\
& 2^{\frac{l\left(J_{\lambda}\right)}{2}}(\sqrt{-1}) \frac{\|\rho\| \|_{J_{\lambda}-m_{J_{\lambda}}}}{\frac{\prod_{\gamma \in J_{\lambda}} \prod_{c \in \Gamma_{*}} z_{\rho_{\gamma}(c)}}{2}}
\end{aligned}
$$

(ii) The first case: if $\rho$ can not be decomposed into $\cup_{\gamma \in \Gamma^{*}}\left(\rho_{\gamma}\right)$ such that $\rho_{\gamma} \in \mathcal{S P} \mathcal{P}_{\left|\lambda_{\gamma}\right|}\left(\Gamma_{*}\right)$, then we have $\chi_{\lambda}^{+}\left(D_{\rho}^{+}\right)=0$. So we can assume that $\rho=\cup_{\gamma \in \Gamma^{*}}\left(\rho_{\gamma}\right)$ such that $\rho_{\gamma} \in \mathcal{S P} \mathcal{P}_{\left|\lambda_{\gamma}\right|}\left(\Gamma_{*}\right)$ and $\chi_{\lambda}^{+}\left(D_{\rho}^{+}\right) \neq 0$.

(1) When $\rho_{\gamma} \in \mathcal{O S} \mathcal{P}_{\left|\rho_{\gamma \mid}\right|}\left(\Gamma_{*}\right)$ for $\gamma \in J_{\lambda}^{\prime}$, we claim that $\rho_{\gamma} \in\left[\lambda_{\gamma}\right]$ for $\gamma \in J_{\lambda}$. In fact, let $S$ be the set of conjugacy classes $\rho$ such that $\rho=$ 
$\cup_{\gamma \in \Gamma^{*}}\left(\rho_{\gamma}\right)$. It is easy to see that if $\xi_{\lambda_{\gamma}}\left(t_{\rho_{\gamma}}\right)$ is nonzero then $\rho_{\gamma}$ must be in $\mathcal{O S P} \mathcal{P}_{\left|\lambda_{\gamma}\right|}\left(\Gamma_{*}\right):=\mathcal{O} \mathcal{P}_{\left|\lambda_{\gamma}\right|}\left(\Gamma_{*}\right) \cap \mathcal{S P}_{\left|\lambda_{\gamma}\right|}\left(\Gamma_{*}\right)$ for $\gamma \in J_{\lambda}^{\prime}$ and $\rho_{\gamma}$ must be in $\left[\lambda_{\gamma}\right]$ for $\gamma \in J_{\lambda}$. Then

$$
\begin{aligned}
& \left\langle\chi_{\lambda}^{+}, \chi_{\lambda}^{+}\right\rangle_{\mathcal{S} \mathcal{P}_{n}^{1}\left(\Gamma_{*}\right)} \geq \sum_{\rho \in S} \frac{1}{\widetilde{Z}_{\rho}}\left|\chi_{\lambda}^{+}(\rho)\right|^{2}=\sum_{\rho \in S} \frac{1}{Z_{\rho}}\left|\chi_{\lambda}^{+}\left(D_{\rho}^{+}\right)\right|^{2} \\
= & \sum_{\rho \in S} K_{\rho}^{2} \frac{1}{\prod_{\gamma \in \Gamma^{*}} 2^{l\left(\rho_{\gamma}\right)} \prod_{c \in \Gamma_{*}} z_{\rho_{\gamma}(c)} \zeta_{c}^{l\left(\rho_{\gamma}(c)\right)}} \cdot \prod_{\gamma \in J_{\lambda}} \prod_{c \in \Gamma_{*}} \gamma(c)^{2 l\left(\rho_{\gamma}(c)\right) .} \\
& \prod_{\gamma \in J_{\lambda}^{\prime}} \prod_{c \in \Gamma_{*}} \gamma(c)^{2 l\left(\rho_{\gamma}(c)\right)} \xi_{\lambda_{\gamma}}^{2}\left(t_{\rho_{\gamma}}\right) \cdot 2^{l\left(J_{\lambda}\right)} \frac{\prod_{\gamma \in J_{\lambda}^{\prime}} \prod_{c \in \Gamma_{*}} z_{\rho_{\gamma}(c)}}{2} \\
\geq & \frac{1}{2} \sum_{\rho \in S} \prod_{\gamma \in J_{\lambda}} \prod_{c \in \Gamma_{*}} \frac{\gamma(c)^{2 l\left(\rho_{\gamma}(c)\right)}}{\zeta_{c}^{l\left(\rho_{\gamma}(c)\right)}} \cdot \prod_{\gamma \in J_{\lambda}^{\prime}} \frac{\prod_{c \in \Gamma_{*}} \gamma(c)^{2 l\left(\rho_{\gamma}(c)\right)} \xi_{\lambda_{\gamma}}^{2}\left(t_{\rho_{\gamma}}\right)}{2^{l\left(\rho_{\gamma}\right)} \prod_{c \in \Gamma_{*}} \zeta_{c}^{l\left(\rho_{\gamma}(c)\right)}} \\
\geq & \frac{1}{2} \prod_{\gamma \in J_{\lambda}}\left(\sum_{\rho_{\gamma} \in\left[\lambda_{\gamma}\right]} \frac{1}{\prod_{c \in \Gamma_{*}} \zeta_{c}^{l\left(\rho_{\gamma}(c)\right)}} \prod_{c \in \Gamma_{*}} \gamma(c)^{2 l\left(\rho_{\gamma}(c)\right)}\right) . \\
& \prod_{\gamma \in J_{\lambda}^{\prime}}\left(\sum_{\rho_{\gamma} \in \mathcal{O} \mathcal{S P}_{\left|\lambda_{\gamma}\right|}\left(\Gamma_{*}\right)} \frac{\prod_{c \in \Gamma_{*}} \gamma(c)^{2 l\left(\rho_{\gamma}(c)\right)} \xi_{\lambda_{\gamma}}^{2}\left(t_{\rho_{\gamma}}\right)}{2^{l\left(\rho_{\gamma}\right)} Z_{\rho_{\gamma}}} .\right. \\
= & \frac{1}{2} \prod_{\gamma \in J_{\lambda}}\left\langle\gamma^{\otimes l\left(\rho_{\gamma}\right)}, \gamma^{\otimes l\left(\rho_{\gamma}\right)}\right\rangle_{\Gamma^{l\left(\rho_{\gamma}\right)}}=\frac{1}{2} .
\end{aligned}
$$

We have known that $\left\langle\chi_{\lambda}^{+}, \chi_{\lambda}^{+}\right\rangle_{\mathcal{S} \mathcal{P}_{n}^{1}\left(\Gamma_{*}\right)}=\frac{1}{2}$, so $\chi_{\lambda}^{+}\left(D_{\rho}^{ \pm}\right)=0$ if $\rho_{\gamma} \notin\left[\lambda_{\gamma}\right]$ for $\gamma \in J_{\lambda}$ and also $K_{\rho}=1$.

(2) If $\rho_{\gamma} \notin \mathcal{O S} \mathcal{S} \mathcal{P}_{\lambda_{\gamma} \mid}\left(\Gamma_{*}\right)$ for $\gamma \in J_{\lambda}^{\prime}$, then there exists one $\rho_{\gamma}$ not in $\mathcal{O} \mathcal{P}_{\left|\lambda_{\gamma}\right|}\left(\Gamma_{*}\right)$ for $\gamma \in J_{\lambda}^{\prime}$. Meanwhile, since $\xi_{\lambda_{\gamma}}$ is a double spin character when $\gamma \in J_{\lambda}^{\prime}$, it is known that it only has nonzero values at even conjugacy classes (i.e. in $\left.\mathcal{O} \mathcal{P}_{n}\left(\Gamma_{*}\right)\right)$. Hence we have $\xi_{\lambda_{\gamma}}\left(t_{\rho_{\gamma}}\right)=0$, therefore $\chi_{\lambda}^{+}\left(D_{\rho}^{ \pm}\right)=0$.

With this result we have determined all remaining character values of the spin characters for $\widetilde{H} \Gamma_{n}$.

\section{References}

[1] G. E. Andrews, Number theory, Dover Publ. Inc., New York, 1994.

[2] D. Benson, Spin modules for symmetric groups, J. London Math. Soc. 38 (1988), 250-262.

[3] I. B. Frenkel, N. Jing and W. Wang. Twisted vertex representations via spin groups and the McKay correspondence. Duke Math. J. 111 (2002), 51-96.

[4] X. Hu and N. Jing. Spin characters of generalized symmetric groups. Monatsh. Math. 173 (2014), 495-518.

[5] X. Hu and N. Jing. Projective irreducible characters of spin wreath products. Proc. Amer. Math. Soc. 143 (2015), 1015-1026.

[6] N. Jing. Vectex operators, symmetric functions, and the spin group $\Gamma_{n} . J$. Algebra 138, (1991), 340-398. 
[7] N. Jing and W. Wang. Twisted vertex representations and spin characters. Math. Zeit. 239 (2002), 715-746.

[8] T. Józefiak. Semisimple superalgebras. In Some Current Trends in Algebra Proc. of the Varna Conf. 1986, Lect. Notes in Math. vol. 1352 (Springer-Verlag, 1988), pp. 96-113.

[9] T. Józefiak. A class of projective presentations of hyperoctahedral group and Schur Q-functions. In Topics in algebra, Banach Center Publication, vol 26 (1990), pp. 317-326.

[10] T. Józefiak. Relating spin representations of symmetric and hyperoctahedal groups. J. Pure and Applied Alg. 7 (2000), 187-193.

[11] I. G. Macdonald. Symmetric functions and Hall polynomials, 2nd ed. (Oxford, 1995).

[12] A. O. Morris. The spin representation of the symmetric group. Proc. London. Math. Soc. 12 (1962), 55-76.

[13] A. O. Morris. Projective representations of Weyl groups. J. London Math. Soc. 8 (2) (1974), 125-133.

[14] A. O. Morris and H. I Jones. Projective representations of generalized symmetric groups. Sém. Lothar. Combin. 50 (2003) Art. B50b, 27 pp. (electronic).

[15] M. Nazarov. Young's symmetrizers for projective representations of the symmetric group. Adv. Math. 127 (1997), no. 2, 190-257.

[16] A. N. Sergeeev. Tensor algebra of the identity representation as a module over Lie superalgebras $G L(n, m)$ and $Q(n)$. Math. USSR Sbornik 51 (1985), no. 2, 419-425.

[17] I. Schur. Über die Darstellung der symmertrischen und der alternierenden Gruppe durch gebrochene lineare Substitutionen. J. Reine Angew. Math. 139 (1911), 155250 .

[18] J. R. Stembridge. The projective representations of the hyperoctahedral group. J. Algebra 145 (1992), 396-453.

[19] M. Yamaguchi. A duality of the twisted group algebra of the symmetric group and a Lie superalgebra. J. Algebra 222 (1999), 301-327.

[20] M. Yamaguchi. A duality of a twisted group algebra of the hyperoctahedral group and the queer Lie superalgebra. In Combinatorial methods in representation theory (Kyoto, 1998), Adv. Stud. Pure Math. vol. 28 (Kinokuniya, Tokyo, 2000), pp. 401422 .

Hu: School of Mathematics and Computer Science, Jianghan University, Wuhan, Hubei 430056, China

E-mail address: xiaolihumath@163.com

Jing: Department of Mathematics, North Carolina State University, Raleigh, NC 27695, USA

E-mail address: jing@math.ncsu.edu 